\title{
Fostering Smart Energy Applications through Advanced Visual Interfaces
}

\author{
Masood Masoodian \\ Department of Computer Science \\ The University of Waikato \\ Hamilton, New Zealand \\ masood@cs.waikato.ac.nz
}

\author{
Saturnino Luz \\ School of Computer Science and Statistics \\ Trinity College Dublin \\ Dublin, Ireland \\ luzs@cs.tcd.ie
}

\author{
Elisabeth André \\ Human Centered Multimedia \\ Augsburg University \\ Augsburg, Germany \\ andre@hcm-lab.de
}

\author{
Thomas Rist \\ Faculty of Computer Science \\ University of Applied Sciences Augsburg \\ Augsburg, Germany \\ thomas.rist@hs-augsburg.de
}

\begin{abstract}
There is an increasing need for technology that assist people with more effective monitoring and management of their energy generation and consumption. In recent years a considerable number of research activities have resulted in a multitude of new ICT-supported tools and services for both the private energy consumer market, as well as for energy related business and industries (e.g., utility and grid companies, facility management, etc.). This workshop focuses on advanced interaction, interface, and visualization techniques for energy-related applications, tools, and services. It brings together researchers and practitioners from a diverse range of background, including interaction design, humancomputer interaction, visualization, computer games, and other fields concerned with the development of advanced visual interfaces for smart energy applications.
\end{abstract}

\section{Categories and Subject Descriptors}

H.5.m. [Information Interfaces and Presentation (e.g. HCI)]: Miscellaneous

\section{General Terms}

Human Factors, Management, Measurement

\section{Keywords}

Energy consumption, energy usage management, energy usage monitoring, information visualization, visual interfaces, user evaluation

This is the author's version of the work. It is posted here for your personal use. Not for redistribution. The definitive Version of Record was published in:

AVI'14, May 27 - 30, 2014, Como, Italy

Copyright 2014 ACM 978-1-4503-2775-6

http://dx.doi.org/10.1145/2598153.2602224

\section{INTRODUCTION}

The global energy consumption is increasing rapidly due to the growing demand around the world. This, coupled with ever-decreasing natural energy resources, is making it necessary to develop more efficient energy-related technologies, not only in terms of consumption, but also in terms of generation and distribution.

To cater for these needs, systems are being developed to allow better monitoring and more effective management of energy generation and consumption technologies, both in domestic and commercial sectors. Examples of these technologies include tools and services such as public displays [8, 1], dashboards $[6,2]$, and virtual environment $[3,7]$ to increase energy-awareness, all kinds of mobile apps for remote energy monitoring and control [5], web-portals to share energy consumption data [4], simulation tools for energy efficient design such as zero-energy buildings, and tools that assist in grid planning tasks. Many of these rely on large collections of data (such as energy consumption data and environmental parameters) and therefore require (or at least could be improved by) using advanced interaction and interface technology to allow smart visual data exploration.

Despite these efforts, however, there is a general lack of coordinated effort in terms of research, as well as development and deployment of advanced visual interfaces for energyrelated applications. For instance many research publications focusing on design, development, and evaluation of energy-related tools and services are often dispersed over a wide range of disciplines. It is therefore becoming important to foster better means of sharing output emerging from research and development efforts through more targeted gatherings and publications focusing on visual interfaces for energy systems.

This workshop aims to fill this existing gap, by focusing on advanced interaction, interface, and visualization techniques for energy-related applications, tools, and services. It bring together researchers and practitioners from a diverse range of background, including interaction design, humancomputer interaction, visualization, computer games, and 
media technology, who are involved in design and development of smart applications in energy-related domains.

\section{TOPICS OF INTEREST}

The workshop aims to include a wide range of topics related to visual interfaces for:

- accessing energy generation and consumption data

- revealing energy-use patterns and behaviour

- relating energy consumption to other information

- energy use analysis

- energy monitoring and management

- energy simulation games

- sharing and comparing energy-use data with others

The workshop also targets research related to user evaluation of such visual interfaces for smart energy applications.

\section{WORKSHOP PLAN}

This one-day workshop will combine short presentations of accepted position papers with long periods of discussions. It will also include a hands-on design exercise session, during which the workshop attendees will be asked to design and sketch a mobile application for providing visual access to the data log of a zero-energy home. The workshop organizers will select the best design from amongst these and award a prize to the winning team.

\section{ACCEPTED POSITION PAPERS}

The workshop includes the following position papers, which cover a diverse range of research related to the topics of the workshop. Papers number 2 and 4 were selected as the best position papers from this workshop, and are published in the AVI 2014 conference proceedings.

1. Watt-a-Feeling - Raising Energy Production Literacy Through a Tangible Installation Filipe Quintal, Clinton Jorge, Valentina Nisi, Nuno Nunes and Mónica Mendes

"Nowadays energy supply is ubiquitous and pervasive. It is everywhere and nowhere. One cannot see or grab hold of energy. This invisibility and intangibility may lead people to misconceive what is energy, thus underestimating its production costs and subsequent financial and environmental impacts. Further, the units of measure of electricity (e.g. kilowatt) are notions difficult to contextualize and understand. In this paper we propose the design of an installation aimed at raising energy production literacy through playful interaction. We propose Watt-a-feeling, a tangible public installation that resorts to the metaphor of an $X$-ray vision of a household wall in an attempt to increase energy literacy by exposing to householders where (which source) their energy is being provided from. Interaction with the installation begins with a visitor producing (locally) enough energy to reveal the $X$-ray vision combined with short comparative facts that should help the public contextualize the production of energy within the larger context of regional energy consumption."
2. Smart Energy Interfaces for Electric Vehicles Paul Monigatti, Mark Apperley and Bill Rogers "Electric vehicle charging strategies rely on knowledge of future vehicle usage, or implicitly make assumptions about a vehicle's usage. For example, a naïve charging strategy may assume that a full charge is required as soon as possible and simply charge at the maximum rate when plugged in, whereas a smart strategy might make use of the knowledge that the vehicle is not needed for a number of hours and optimise its charging behaviour to minimise its impact on the electricity grid. These charging strategies may also offer vehicle-to-grid services. To achieve this functionality, a driver needs to specify the details of the next trip-or sequence of trips - in order for the charging strategy to perform optimally. This paper explores the value of next-trip information, and presents a potential user interface to assist a driver with providing these details."

3. Advanced Visual Interfaces for Smart Energy: Focusing Where it Matters Most

Kent Wittenburg, Christopher Laughman, Daniel Nikovski and Zafer Sahinoglu

"Targeting reductions of home electricity usage among consumers in their homes has been very popular among researchers, industry, and research funding organizations, but we argue that commercial and industrial applications may have the best chance of achieving significant environmental impact. We support this position through an analysis of energy resources consumed by different sectors in the US and observations regarding economic incentives and leverage. We then give examples of industrial and commercial energy-savings applications being developed at Mitsubishi Electric and supported by its North American Research Laboratory, MERL."

4. Interactive Visual Tools for the Planning and Monitoring of Power Grids

Thomas Rist and Michael Wiest

"In this contribution we argue that power grid design and monitoring is an application domain that could greatly benefit from novel visualization techniques as well as from advances in interactive graphics. We refer to some selected works to support our point of view."

5. Towards using Exploratory Sequential Data Analysis for Smart Buildings

Simon Breslav, Alex Tessier, Ramtin Attar and Azam Khan

"Buildings have been identified as being the largest cause of greenhouse gas production in the world (48\%) due to over-cooling, over-heating, and over-lighting. To help reduce these inefficiencies in buildings, a growing number of data analytics startups in the energy sector are working with big data and machine-learning algorithms in the cloud that will ultimately save energy for building owners and improve the sustainability of their operations. We endeavor to look beyond performing pattern recognition in the cloud by creating a solid foundation of advanced visual interfaces that support Exploratory Sequential Data Analysis (ESDA) for Building Information Modeling (BIM)." 
6. Evaluating the Effectiveness of Visualizations for Comparing Energy Usage Data

Elisabeth André, René Bühling, Birgit Endrass and Masood Masoodian

"In recent years, various interactive systems and visualization techniques have been proposed to promote energy saving by encouraging people to compare their energy usage data with those of others, as well as with their own historical usage data. Many of these systems rely on conventional visualizations such as time-series and pie charts to allow making such comparisons. Unfortunately however, most of these visualizations have never been evaluated to assess their effectiveness in allowing easy comparisons of different energy usage data. In this paper we discuss the need for formal evaluation of visualizations that aim to support such comparisons, and provide a case study using an evaluation we have conducted to compare two alternative visualizations that we have developed."

7. Pervasive Visual Interfaces to Change Energy Consumption Behaviour at the Workplace Dirk Börner, Marco Kalz, Stefaan Ternier and Marcus Specht

"This position paper introduces pervasive interventions at a university campus to increase the pro-environmental awareness, consciousness, and learning of employees making use of different visual interfaces. We briefly present the design of three intervention iterations. While in the first intervention the focus was on increasing awareness through information distribution with ambient learning displays on the campus, the second iteration provided personalised feedback to employees with the help of a sensor network and different client applications. The third iteration then implemented a game-based learning concept. We found that these approaches are effective on different levels and that a combination of these effective elements can lead to a sustained behaviour change among the employees."

8. Why aren't we all living in Smart Homes? Joris Suppers and Mark Apperley

"Visions of the Future, like the Jetsons cartoons, show homes which are smart and able to control household appliances, to make living easier and more comfortable. Although much research has been carried out into the effectiveness of different visualization techniques for conveying useful energy consumption information to householders, and in techniques for controlling the timing and coordination of appliance use, these techniques have failed to achieve widespread penetration, and the vision still seems far from a reality. This paper examines the reasons why smart home technologies have so far failed to have any real impact, and we are not all living in them. It examines this question under four sections: Technology, Consumers, Electricity retailers and Government agencies, using examples from New Zealand's electricity sector."

9. Towards a More Responsible Use of Energy Through Visualization of Energy Data Thomas Rist

"This contribution recalls some general objectives, prerequisites and assumptions related to the responsible use of energy. We work through a number of different approaches that use visualizations of energy-related data. The paper aims to stimulate discussion on the effectiveness of different visualization types with regard to objectives related to a more responsible use of energy."

10. Visualizing a Control Strategy for Estimating Electricity Consumption

Patrick Ozoh, Shapiee Abd-Rahman and

Mark Apperley

"This paper investigates the potential of applying different control measures on low power and high power appliances with the goal of evolving efficiency in electricity consumption. The research involves carrying out simulations on their power consumption readings to set up a control system. The study discovers savings on all appliances under study to be $12.8 \% \mathrm{Kw}$, not minding occupancy rate of the building. Air-conditioners have the greatest impact of a $6 \% \mathrm{Kw}$ contribution on savings. This would lead to a substantial contribution when converted to pricing rates. The results from the study indicate that control measures should be extended to peak periods and power saving measures extended to more appliances."

11. The Social Power Game: A Smart Application for Sharing Energy-saving Behaviours in the City Vanessa De Luca and Roberta Castri

"In this paper, we introduce Social Power Game, a mobile game application that aims at encouraging energy saving through social interaction. Instead of an individual energy analytic approach, this game incorporates social interaction and gamification as crucial principles for curbing energy consumption, fostering community collaboration and increasing people's intrinsic motivation. Based on an interdisciplinary research conducted in Switzerland, this paper describes motivations, design and interaction mechanisms for the application."

\section{DISSEMINATION}

Accepted position papers will be published in the workshop proceedings. The workshop participants will receive a copy of the proceedings, which will also be made available through the workshop website ${ }^{1}$. The workshop will include a discussion on publication of the extended versions of the position papers in the form of, for instance, a special issue of a journal, or an edited volume.

\section{ORGANIZERS}

Masood Masoodian is a senior lecturer in the Department of Computer Science at the University of Waikato. His research interests include visualization and interaction design, specializing in visualization of time-based data (e.g. energy and health-related data). He has been involved in design, development, and evaluation of numerous visual interfaces and visualization systems for various technology ranging from large interactive displays to small mobile devices. He has

\footnotetext{
${ }^{1}$ http://hcm-lab.de/projects/AVI2014/
} 
served as the programme chair, programme committee member, and reviewer for many international conferences and scientific journals.

Elisabeth André is a full professor in Computer Science, and the Chair of Human-Centered Multimedia in the Institute for Informatics at Augsburg University. She has been involved in organization of numerous international conferences, serving as Programme Co-Chair of Autonomous Agents 2001, Programme Co-Chair of International Conference on Intelligent User Interfaces 2003, General Chair of Fourth German Conference on Multiagent System Technologies 2006, General Conference Co-Chair of 7th International Conference on Intelligent Virtual Agents 2007, Program Co-Chair of the 23rd Annual Conference on Computer Animation and Social Agents 2010, Program Co-Chair of International Conference on Intelligent User Interfaces 2011, Program Co-Chair of 4th International Conference on Interactive Digital Storytelling 2011. She is also Associate Editor of IEEE Transactions on Affective Computing (TAC), Associate Editor of ACM Transactions on Intelligent Interactive Systems (TIIS), Member of the Editorial Board of the Journal of Autonomous Agents and Multi-Agent Systems (JAAMAS), Member of the Editorial Board of Journal on Multimodal Interfaces, Member of the Editorial Board of AI Communications (AICOM), and was Associate Editor of International Journal of Human-Computer Studies from 2007 to 2010

Saturnino Luz is a lecturer in the School of Computer Science and Statistics). He graduated in Computer Science and holds a PhD degree in Informatics. His research spans the areas computer supported cooperative work, information visualization, natural language processing, dialogue and multimedia systems. He has participated in a number of national and EU-funded research projects, working on intelligent information interfaces and human language technology. His work includes an in-depth investigation of human interaction at meetings, with particular focus on medical team meetings. He is a member of the Association for Computing Machinery (ACM) and contributes regularly to the ACM Computing Reviews.

Thomas Rist is a full professor in Computer Science at the University of Applied Sciences Augsburg. He has a long track-record in the field of intelligent user interfaces, and interactive media systems. His current research activities comprise interdisciplinary work at the intersection of HCI and energy-related applications. He has coordinated a number of EU and nationally funded research projects, and is currently the coordinator of the German-NZ research network IT4SE (see the Acknowledgements section). He has served as a PC or OC member of various national and international scientific workshops, symposia, and conferences.

\section{PROGRAM COMMITTEE}

Workshop position papers were selected through a singleblind reviewing process. We would like to thank the members of our program committee:

Elisabeth André, Augsburg University.

Mark Apperley, The University of Waikato.

Matt-Mouley Bouamrane, University of Glasgow.

Luciano Gamberini, University of Padova.

Giulio Jacucci, University of Helsinki.

Cecilia Katzeff, Interactive Institute Swedish ICT.

Roni Khardon, Tufts University.
Saturnino Luz, Trinity College Dublin.

Masood Masoodian, The University of Waikato.

Thomas Rist, University of Applied Sciences Augsburg.

Bill Rogers, The University of Waikato.

Manfred Tscheligi, University of Salzburg.

Kent Wittenburg, MERL.

\section{ACKNOWLEDGEMENTS}

This workshop is supported by the IT4SE project, funded by the German Federal Ministry of Education and Research (Grant number NZL 10/803 IT4SE) under the APRA initiative. More information about the IT4SE project can be found at http://www.it4se.net.

\section{REFERENCES}

[1] Bird, J., and Rogers, Y. The pulse of tidy street: Measuring and publicly displaying domestic electricity consumption.

http://www. changeproject.info/publications/ energyAwarenessWorkshopBirdRogers .pdf, 2014. Last accessed in April 2014.

[2] Delta Controls. Earthright energy dashboard. http://www.deltacontrols.com/products/ energy-management/energy-awareness/ earthright-energy-dashboard, 2014. Last accessed in April 2014.

[3] Gouveia, L., Pereira, L., Scott, M., and Oakley, I. Eco-Avatars: visualizing disaggregated home energy use. http://www.m-iti.org/uploads/u117/Gouveia_ Pereira_Scott_Dakley-DIS-2012.pdf, 2012. Last accessed in April 2014.

[4] Kamilaris, A., Trifa, V., and Guinard, D. Building web-based infrastructures for smart meters. http://seacorn.cs.ucy.ac.cy/papers/files/ KamilarisPervasive10.pdf, 2014. Last accessed in April 2014.

[5] Kugler, M., André, E., Masoodian, M., Reinhart, F., Rogers, B., and Schlieper, K. Assisting inhabitants of residential homes with management of their energy consumption. In Sustainability in Energy and Buildings, A. Hakansson, M. Höjer, R. J. Howlett, and L. C. Jain, Eds., vol. 22 of Smart Innovation, Systems and Technologies. Springer Berlin Heidelberg, 2013, 147-156.

[6] Schneider Electric. Use one dashboard to drive your energy awareness and business objectives. http://www. powerlogic.com/literature/ 3000H00603R1108_IONDemand.pdf, 2009. Last accessed in April 2014.

[7] Shiraishi, M., Washio, Y., Takayama, C., Lehdonvirta, V., Kimura, H., and Nakajima, T. Using individual, social and economic persuasion techniques to reduce co2 emissions in a family setting. In Proceedings of the 4th International Conference on Persuasive Technology, Persuasive '09, ACM (New York, NY, USA, 2009), 13:1-13:8.

[8] Valkanova, N., Jorda, S., Tomitsch, M., and Vande Moere, A. Reveal-it!: The impact of a social visualization projection on public awareness and discourse. In Proceedings of the SIGCHI Conference on Human Factors in Computing Systems, CHI '13, ACM (2013), 3461-3470. 